\title{
LOBECTOMY COMBINED WITH VOLUME REDUCTION FOR PATIENTS WITH LUNG CANCER AND ADVANCED EMPHYSEMA
}

Steven R. DeMeester, MD* G. Alexander Patterson, MD

R. Sudhir Sundaresan, MD

Joel D. Cooper, MD

Objective: Early-stage lung cancer is best treated by anatomic pulmonary resection. Patients with lung cancer and severe emphysema are often denied resection or are offered only limited, nonanatomic resections when established pulmonary function criteria for lobectomy are not met. Recently, with the introduction of the volume reduction operation, selected patients with disabling emphysema have undergone excision of approximately $30 \%$ of the most destroyed lung tissue and have subsequently demonstrated subjective and objective improvement in pulmonary function. Using these principles, we elected to combine anatomic lobectomy with volume reduction in a select group of patients with both emphysema and lung cancer who would not otherwise be candidates for pulmonary resection. Methods: Five patients with severe emphysema and suspected or proven lung cancers, who were poor candidates for anatomic lobectomy by traditional criteria but were good candidates for volume reduction, underwent lobectomy combined with volume reduction of one or more additional lobes. Results: All five patients having lung volume reduction and anatomic lobectomy for early-stage primary lung cancer did well postoperatively. Furthermore, each patient has demonstrated subjective and objective improvement in respiratory function on serial postoperative studies. Conclusions: Selected patients with disabling emphysema and suitable anatomy for volume reduction, who have a lung cancer situated in destroyed lung tissue, may benefit from combined lobectomy and volume reduction. The introduction of the volume reduction operation has added a new factor in the algorithm for the evaluation and treatment of lung cancer in selected patients with advanced emphysema. (J Thorac Cardiovasc Surg 1998;115: 681-8)

Early-stage non-small-cell lung cancer is best treated Eby surgical resection. Ideally, the resection should consist of either lobectomy or pneumonectomy. Limited resection with wedge excision or segmentectomy

From the Division of Cardiothoracic Surgery, Department of Surgery, Washington University School of Medicine, BarnesJewish Hospital, St. Louis, Mo., and (*current address) the Division of Cardiovascular and Thoracic Surgery, Department of Surgery, University of Southern California, Los Angeles, Calif.

Received for publication April 10, 1997; revisions requested July 15, 1997; revisions received Oct. 16, 1997; accepted for publication Oct. 16, 1997.

Address for reprints: Joel D. Cooper, MD, Division of Cardiothoracic Surgery, Washington University School of Medicine, One Barnes Hospital Plaza, 3108 Queeny Tower, St. Louis, MO 63110

Copyright (C) 1998 by Mosby, Inc.

0022-5223/98 \$5.00+ $0 \quad \mathbf{1 2 / 1 / 8 7 0 4 9}$ has been associated with a significantly increased risk of local recurrence and a reduction in both overall and disease-free survival. ${ }^{1}$ Occasionally though, associated emphysema in patients with lung cancer so compromises pulmonary function as to preclude resection. For such patients alternative treatment strategies including radiation therapy, with or without chemotherapy, have yielded comparatively poor results. ${ }^{2,3}$ Furthermore, high-dose radiation may lead to as much reduction in pulmonary function as a surgical resection. Thus limited pulmonary function in patients represents as much a management problem for the radiation oncologist as for the surgeon. ${ }^{4}$ This dilemma is further compounded for surgeons by the lack of a precisely definable point at which the risk-to-benefit ratio for resection becomes unfavorable, particularly in light of improvements in the anesthetic, surgical, and postoperative treatment of patients with advanced emphysema. 

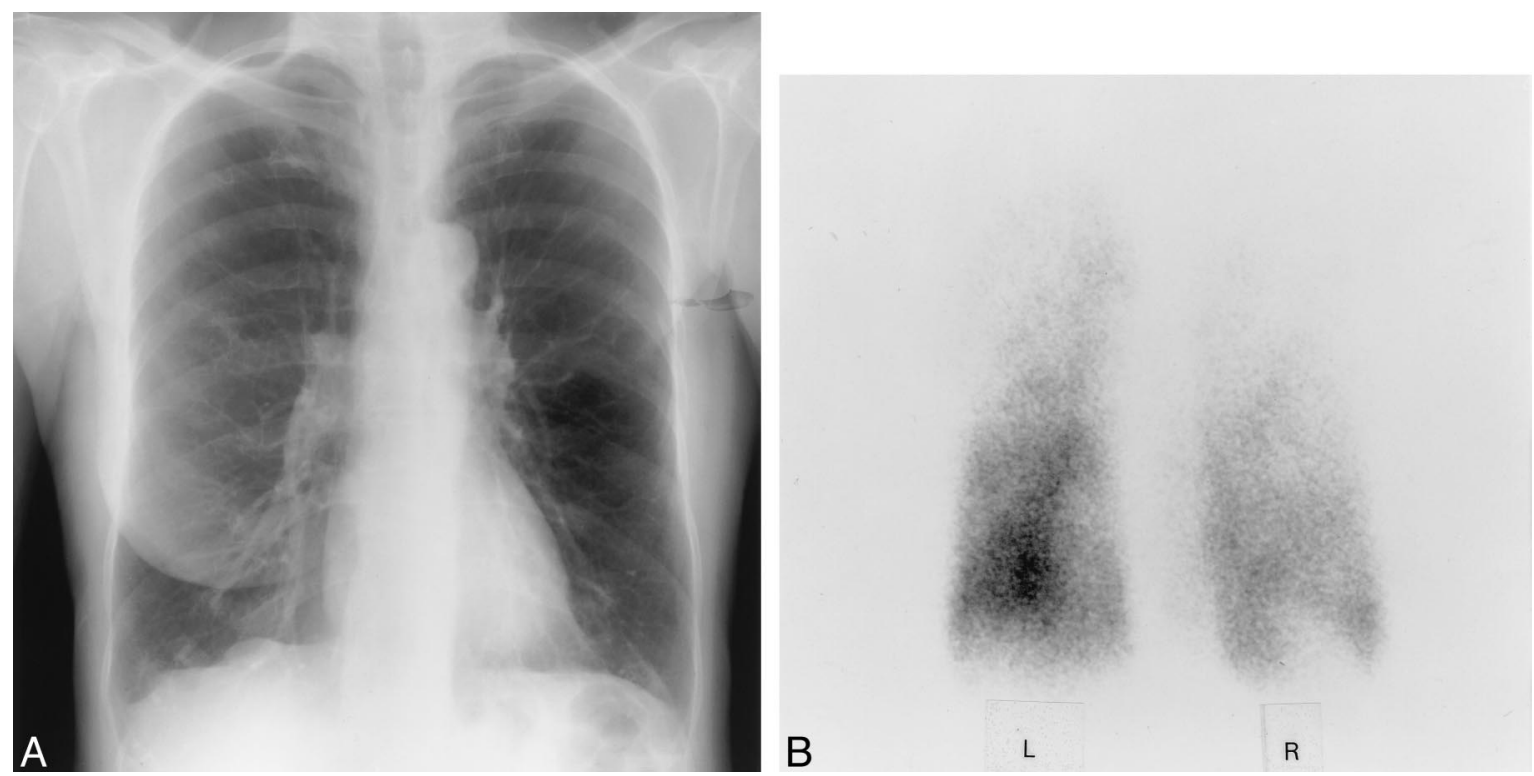

Fig. 1. A, Posteroanterior chest $x$-ray film of patient a showing characteristics of severe emphysema including bilateral pulmonary hyperinflation and flattened diaphragms. The right upper lobe nodule is faintly visible. B, Posterior view of the ventilation portion of a ventilation-perfusion scan from the same patient. The left lung has greater overall ventilation, and there is bilateral upper lobe destruction, particularly on the right.

We have previously reported the beneficial early and intermediate-term results of lung volume reduction (LVR) in 150 selected patients with advanced emphysema. ${ }^{5,6}$ In $10 \%$ of these patients a complete, anatomic lobectomy was included in the bilateral procedure because the entire lobe was destroyed by emphysema and the anatomic findings at operation were favorable for a lobectomy. Interestingly, pathologic examination of resected lung tissue in 2 of the 150 patients revealed a focus of cancer not detected preoperatively. Subsequently, five patients were seen with suspected or proven lung cancer and severe emphysema anatomically amenable to LVR. In each patient the respiratory compromise from emphysema was so severe that under previous selection criteria none of them would have been considered suitable candidates for a major pulmonary resection. Furthermore, in each patient the lung cancer was situated such that wedge excision would be difficult or impossible. However, given the demonstrated benefits of LVR in patients with a suitable anatomic situation, and the lack of satisfactory alternative treatment in these select patients with lung cancer, we chose to perform a cancer resection with anatomic lobectomy in conjunction with LVR. The purpose of this report is to describe these cases and the early postoperative results.

\section{Methods}

Between October 1994 and October 1996, 608 patients with severe emphysema were evaluated at Barnes Hospital as potential candidates for LVR operations. Among these 608 patients, 142 were determined to be acceptable candidates and went on to have LVR operations. Among the 142 patients accepted for operation were three patients $(2.1 \%)$ in whom chest $\mathrm{x}$-ray films showed a pulmonary nodule suspicious for carcinoma. Another two patients with severe emphysema were referred for evaluation of newly diagnosed lung cancers. These five patients (patients a through e) form the basis for this report.

The mean age of the five patients was 62 years (range 53 to 70 years), and three were men. None of the patients had symptoms from the cancer, but all had symptoms of advanced emphysema. Two patients had dyspnea at rest (patients $\mathrm{c}$ and $\mathrm{d}$ ) and required continuous supplemental oxygen. One patient (patient d) was receiving $30 \mathrm{mg} /$ day of prednisone when first evaluated but was weaned to 5 $\mathrm{mg}$ /day before operation. A preoperative diagnosis of cancer was made in four patients (all except patient a). Evaluation in each patient included inspiratory and expiratory posteroanterior and lateral chest x-ray films, chest computed tomographic scans including views of the liver and adrenal glands, and a ventilation/perfusion scan. A representative chest $\mathrm{x}$-ray film and a ventilation/perfusion scan from one patient (patient a) are shown in Fig. 1. Candidates for combined lobectomy and volume reduction had to have radiographic and scintigraphic evidence of severe emphysema in a heterogeneous distribution. Furthermore, the pulmonary nodule had to be located in 
Table I. Results of pulmonary function, arterial blood gas, and 6-minute walk tests for the five patients at the time they were accepted for surgical treatment

\begin{tabular}{|c|c|c|c|c|c|}
\hline & \multicolumn{5}{|c|}{ Patient } \\
\hline & $a$ & $b$ & $c$ & $d$ & $e$ \\
\hline FVC (L) & $1.98(70)^{*}$ & $3.12(71)$ & $2.00(74)$ & $3.13(76)$ & $2.80(74)$ \\
\hline $\mathrm{FEV}_{1}(\mathrm{~L})$ & $0.64(28)$ & $1.14(33)$ & $0.67(33)$ & $0.85(24)$ & $0.90(30)$ \\
\hline $\mathrm{FEV}_{25-75}(\mathrm{~L} / \mathrm{sec})$ & $0.20(9)$ & $0.40(12)$ & $0.23(10)$ & $0.21(5)$ & $0.15(5)$ \\
\hline $\operatorname{TLC}(\mathrm{L})$ & $6.86(138)$ & $11.6(160)$ & $6.21(133)$ & $9.26(129)$ & $9.26(145)$ \\
\hline RV (L) & $4.88(232)$ & $8.43(340)$ & $4.15(221)$ & $6.06(287)$ & $6.40(308)$ \\
\hline $\mathrm{DLCO}_{\text {unc }} \%$ & 44 & 37 & 43 & 23 & 44 \\
\hline $\mathrm{PaO}_{2}(\mathrm{~mm} \mathrm{Hg})(\mathrm{RA}) \dagger$ & 64 & 59 & 72 & 38 & 77 \\
\hline $\mathrm{PaCO}_{2}(\mathrm{~mm} \mathrm{Hg})$ & 33.7 & 37.5 & 36 & 54 & 42 \\
\hline 6-Min walk (feet) & 1100 & NA & 600 & 40 & 1800 \\
\hline
\end{tabular}

$F V C$, Forced vital capacity; $T L C$, total lung capacity; $R V$, residual volume; $D L C O_{u n c} \%$, uncorrected diffusing capacity as a percentage of predicted; $P a O_{2}$, arterial oxygen tension; $\mathrm{PaCO}_{2}$, arterial carbon dioxide tension.

*The number in parenthesis is the percent of predicted for that patient.

$\dagger \mathrm{RA}$ refers to inspired oxygen concentration equal to room air.

Table II. Location of the tumor, operative procedure performed, pathologic features including stage, and postoperative discharge day for each of the five patients

\begin{tabular}{ccllc}
\hline Patient & Tumor location & \multicolumn{1}{c}{ Operative procedure } & \multicolumn{1}{c}{ Pathology } & Postop. discharge day \\
\hline a & RUL & RU lobectomy with LUL reduction & Adenocarcinoma, stage I & 7 \\
b & RML & RM lobectomy with bilateral UL reduction & Adenocarcinoma, stage I & 13 \\
c & RML & RM lobectomy with RUL reduction & Adenocarcinoma, stage I & 24 \\
d & LUL & Staged RU lobectomy, LU lobectomy & Adenocarcinoma, stage I & $9 / 12$ (first/second operation) \\
e & LUL & LU lobectomy with RUL reduction & Squamous cell, stage II & 12
\end{tabular}

$R U L$, Right upper lobe; $L U L$, left upper lobe; $R M L$, right middle lobe; $U L$, upper lobe.

one of the more destroyed lobes. Evaluation of each patient also included pulmonary function tests (PFTs), an arterial blood gas, and a 6-minute walk test (Table I). Systemic staging consisted of a brain computed tomogram and radionucleotide bone scan in each patient. A noninvasive cardiac evaluation was done before the operation as a standard procedure. One patient (patient e) was found to have coronary artery disease and ultimately required angioplasty before undergoing pulmonary resection. In another patient (patient c), a prior myocardial infarction resulted in a reduced left ventricular ejection fraction (26\%), but no areas of active ischemia were identified. No other nonpulmonary organ dysfunction was identified in these patients.

Before resection four of the five patients underwent 6 to 8 weeks of supervised pulmonary rehabilitation. One patient (patient b), in whom the lung cancer was discovered on a chest x-ray film done in an emergency department after a biking accident, was in good physical condition and did not undergo preoperative rehabilitation. In another patient (patient d), the pulmonary nodule became apparent after rehabilitation, before the planned LVR operation.

At operation, each patient underwent bronchoscopy, and four of the five patients had mediastinoscopy. One patient (patient a) had a small, undiagnosed right upper lobe lung nodule with no computed tomographic evidence of mediastinal adenopathy, and she did not undergo mediastinoscopy. A median sternotomy was used in four of the five patients. One patient (patient d) had previously undergone bleb excision and pleurectomy on the left side because of recurrent spontaneous pneumothorax, and he underwent bilateral staged thoracotomies. Pleural tents were used to eliminate residual intrathoracic space after resection in two of the five patients. The operative procedure and pathologic stage of the resected tumor for each patient are shown in Table II.

Postoperative pain management for each patient consisted of a thoracic epidural catheter and a combination of narcotic and local anesthetic. Each patient was extubated in the operating room and taken to the thoracic observation (step-down) unit after a short stay in the recovery room. Chest physiotherapy was initiated on the day of the operation and continued throughout the hospital stay. Ambulation and pulmonary rehabilitation began on the first postoperative day. Postoperative bedside bronchoscopy and a minitracheotomy procedure were used when necessary. Patients were discharged from the hospital after removal of the last chest tube (range 7 to 24 days after the operation, mean 12.8 days).

\section{Results}

Preoperative structured pulmonary rehabilitation was used in four of the five patients. There was no significant difference in any PFT parameter after rehabilitation in these four patients. However, the two most deconditioned patients (patients $\mathrm{c}$ and d) 


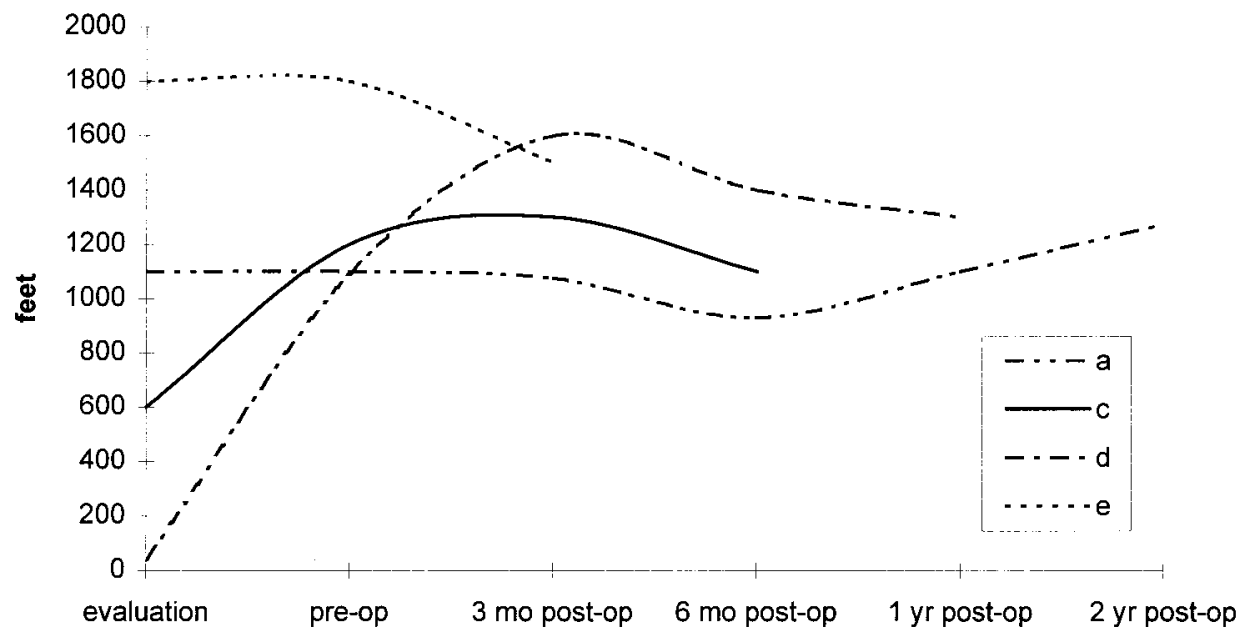

Fig. 2. Distance walked in 6 minutes in each of the four patients who underwent preoperative pulmonary rehabilitation. The greatest gains were demonstrated in the patients most deconditioned at the time of evaluation (patients $\mathrm{c}$ and $\mathrm{d}$ ). The best-conditioned patients at the time of evaluation (patients a and e) did not improve with preoperative structured pulmonary rehabilitation and showed early postoperative decline in 6-minute walk distance.

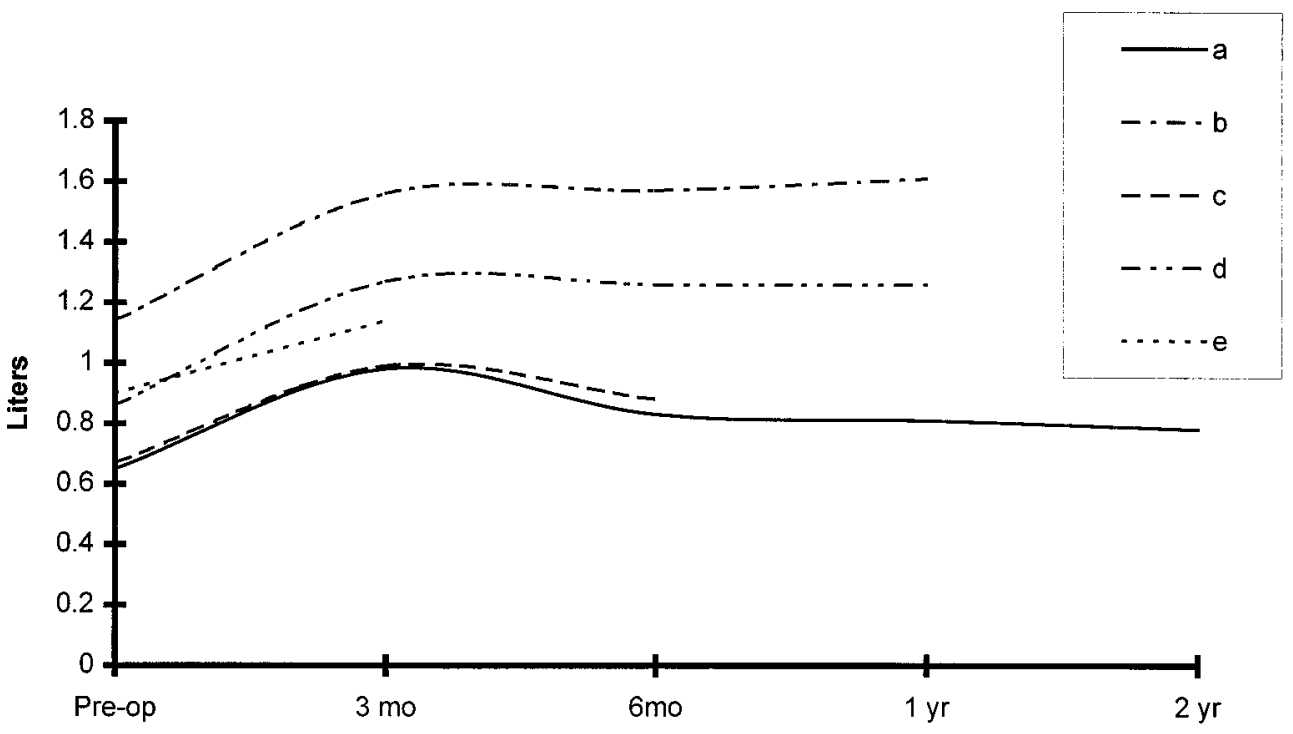

Fig. 3. $\mathrm{FEV}_{1}$ values improved in each patient after lobectomy and volume reduction when compared with the postrehabilitation, preoperative value. The improvements have persisted for 2 years in one patient and for 1 year in two patients. Peak postoperative improvement seems to occur during the first 6 months and is followed by a slow decline toward the preoperative value (see discussion). Importantly, note that the postoperative $\mathrm{FEV}_{1}$ value was not decreased after lobectomy and volume reduction in any of the five selected patients with advanced emphysema and lung cancer.

demonstrated substantial improvement in 6-minute walk distance after rehabilitation as shown in Fig. 2. These two patients continued to show improvement in 6-minute walk distance in the postoperative period. Interestingly, the two patients who were better conditioned at evaluation (patients a and c) demonstrated no improvement with rehabilitation and covered less distance in the early postoperative period than they had immediately before the operation. With longer follow-up, however, the 6-minute 


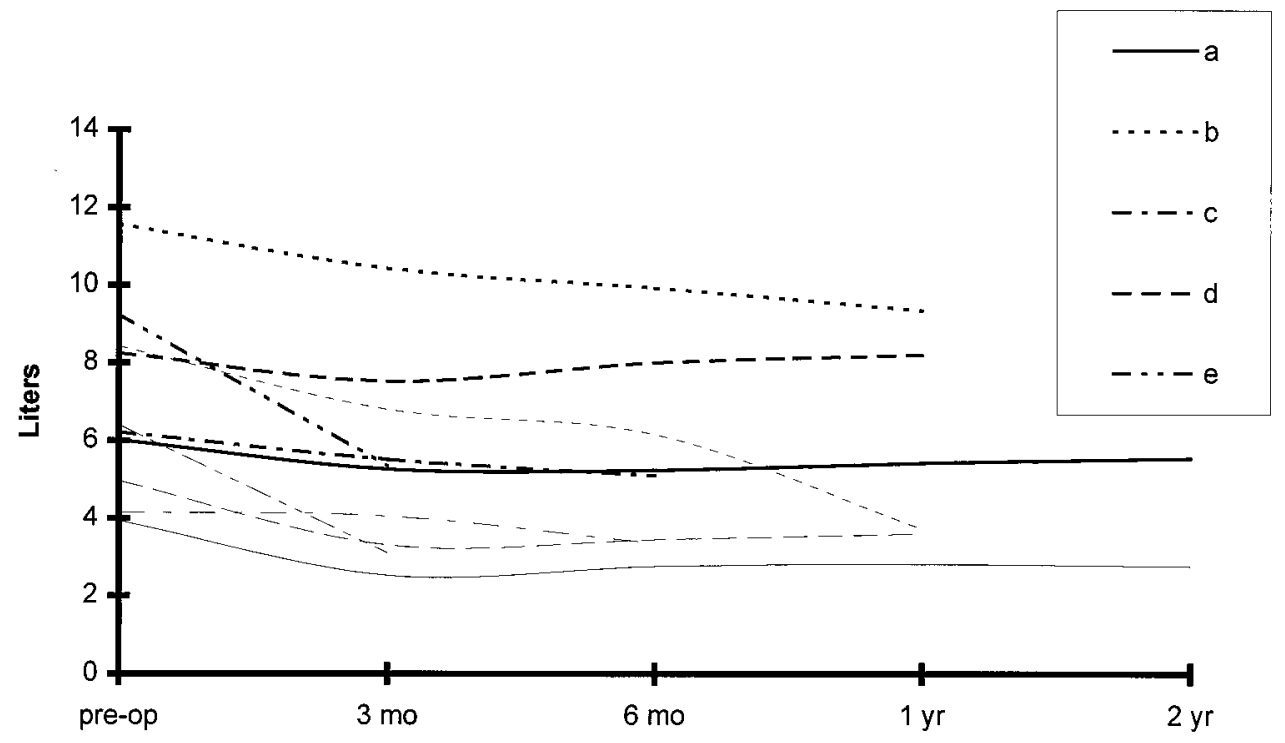

Fig. 4. Both total lung capacity (TLC, shown in bold lines) and residual volume ( $R V$, shown in light lines) decreased after lobectomy and volume reduction in selected patients with advanced emphysema and lung cancer.

walk distance has steadily improved in the one patient with a 2-year follow-up after resection.

Despite the fluctuations in postoperative 6-minute walk distance, each patient has had significant subjective and objective improvement in pulmonary function. Measurable improvements in the first-second forced expiratory volume $\left(\mathrm{FEV}_{1}\right)$, total lung capacity, and residual volume were seen in all five patients as shown in Figs. 3 and 4. These improvements have persisted for 2 years after the operation in one patient (patient a), albeit with a slow, steady return toward the preoperative values. Maximal postoperative improvement in $\mathrm{FEV}_{1}$ averaged $43 \%$ in the five patients, with a range from $27 \%$ to $51 \%$. Importantly, in no patient did the $\mathrm{FEV}_{1}$ decrease after resection. In addition to the improvement in pulmonary function, each patient has benefited from optimal treatment for the lung cancer. In four of the five patients the lung cancer was found to be stage I and they required no further therapy. The one patient with a stage II cancer (patient e) received postoperative adjuvant radiation therapy. During a mean follow-up of 19 months there has been no evidence of new or recurrent cancer in any of the patients.

There was no mortality; however, three of the five patients had complications. One patient's hospitalization (patient b) was prolonged as a consequence of bronchitis and retained airway secretions necessitating bedside bronchoscopy. A pneumothorax de- veloped in one patient (patient c) after chest tube removal and in combination with retained airway secretions produced respiratory distress necessitating intubation and mechanical ventilation. Bronchoscopy and chest tube placement were performed, and the patient was successfully extubated less than 24 hours after intubation. However, a prolonged air leak from the new chest tube delayed the patient's discharge from the hospital. Both of the patients with pleural tents (patients $b$ and e) had postoperative bleeding into the extrapleural space created by the pleural tent. Blood transfusion and placement of a chest tube into the intrathoracic, extrapleural space were required in each patient. In both patients the chest tube adequately decompressed the extrapleural space, and no further therapy was required.

\section{Discussion}

Cigarette smokers are at increased risk for both emphysema and lung cancer. ${ }^{7,8}$ In a review from the University of Pittsburgh, $4.8 \%$ of patients evaluated for LVR operations were found to have an unsuspected lung lesion. Of these lesions, $60 \%$ proved to be primary lung cancers. ${ }^{9}$ Although surgical resection with an anatomic lobectomy or pneumonectomy has been demonstrated to be the optimal treatment for patients with early-stage lung cancer, the presence of significant emphysema has elimi- 
nated many patients from consideration for major pulmonary resection.

A number of studies have examined the relationship of preoperative PFT findings and operative outcome in an attempt to predict perioperative risk for a patient requiring pulmonary resection and to define criteria for inoperability. In 1971 Boushy and associates ${ }^{10}$ reported that of all the PFT values, $\mathrm{FEV}_{1}$ was the most helpful in selecting patients at high risk for postoperative mortality. Miller, Grossman, and Hatcher ${ }^{11}$ in 1981 and Miller ${ }^{12}$ in 1993 published findings from a series of 1831 pulmonary resections, including 785 lobectomies and 161 pneumonectomies, which were done with an overall operative mortality rate of $0.65 \%$. In this series, the minimum criteria for lobectomy were a maximal voluntary ventilation greater than $40 \%$ of predicted, an $\mathrm{FEV}_{1}$ value greater than $1 \mathrm{~L}$, and an $\mathrm{FEV}_{25-75}$ value of greater than $0.6 \mathrm{~L}$. Ferguson and colleagues,${ }^{13}$ on the other hand, analyzed PFT data from 237 patients who underwent pulmonary resection and determined that the most important predictor of death and pulmonary complications was the corrected diffusing capacity as a percentage of predicted. They recommended that patients in whom this value was less than $60 \%$ be excluded from consideration for major pulmonary resection.

In an effort to further define the operative risk in patients with marginal PFT findings, Olsen and Block ${ }^{14}$ Kristersson, ${ }^{15}$ Ali, ${ }^{16}$ Wernly, ${ }^{17}$ and their associates have reported on methods to quantify differential lung function on the basis of xenon 133 ventilation radiospirometry with or without technetium 99 perfusion scanning. In the series by Wernly and coworkers, ${ }^{17}$ patients with a predicted postresection $\mathrm{FEV}_{1}$ value of less than $1 \mathrm{~L}$ were considered unfit for resection. Others have suggested that the loss of pulmonary vascular compliance or an impaired maximal oxygen consumption during exercise will help define patients at high risk for resection.

Despite the multitude of methods to assess perioperative risk for resection, the fact remains that no single test, number, or percentage has been demonstrated to reliably separate patients who will tolerate resection from those who will not. Rather, most thoracic surgeons rely on several of these tests, in addition to the overall physical state of the patient, to select operative candidates. With the development of LVR operations, however, patients with advanced emphysema who do not meet traditional PFT and clinical criteria for pulmonary resection have undergone resection of $20 \%$ to $40 \%$ of the volume of each lung and have shown remarkable postoperative improvement in both symptoms and measured pulmonary function. ${ }^{18}$ Postulated mechanisms include an improvement in elastic recoil of the lung; reduced airway resistance; an improvement in diaphragmatic function, chest wall mechanics, and respiratory muscle efficiency; and an overall reduction in ventilation/perfusion mismatch. ${ }^{18-20}$

Having established a successful volume reduction program, and having gained experience with the perioperative treatment of patients with advanced emphysema, it seemed logical to extend the benefits of the volume reduction concept to a select group of patients with the combination of early-stage lung cancer and severe emphysema. Patients whose disease might otherwise be considered inoperable could potentially undergo an optimal cancer operation with anatomic lobectomy and simultaneously achieve an overall improvement in respiratory status. This is precisely what has occurred in this early series of patients, each of whom had PFT values that in one or more categories fell well below the traditionally accepted minimal criteria for lobectomy. Consistent with reports on patients who have undergone volume reduction operation alone, each of these patients had postoperative improvement in the PFT findings, with an increase in $\mathrm{FEV}_{1}$ and a decrease in the total lung capacity and residual volume. These objective improvements in measured pulmonary function correlated with improvement in each patients' symptoms of dyspnea and with an improvement in the 6-minute walk distance in four of the five patients.

It is important to recognize that in these selected patients, as a consequence of the heterogeneous distribution of the emphysema, anatomic lobectomy was accomplished without any patient having a decrease in $\mathrm{FEV}_{1}$ values after resection. Furthermore, the PFT improvements have persisted in the postoperative period, although a slow return toward the preoperative values is apparent in the one patient with 2-year follow-up. This steady decline likely represents the natural history of severe emphysema, with gradual deterioration of the remaining emphysematous lung tissue. This finding reinforces the concept that volume reduction operations may "set the clock back" for patients with advanced emphysema but do not cure them of the disease.

As with any volume reduction operation, we believe that a combined cancer and volume reduction operation should be offered only to a very select 
group of patients. Specifically, ideal candidates for a combined operation would have symptomatic emphysema with hyperinflated lungs and target areas of severe destruction, with the cancer located in a target lobe. Two of our patients were not ideal in that the cancer was in the middle lobe, whereas the most destroyed lung was in the upper lobes. However, middle lobectomy in combination with upper lobe volume reduction was well tolerated in each patient despite the loss of some functioning middle lobe lung parenchyma. In some patients a lobectomy alone may suffice both for the cancer operation and the volume reduction effect. Indeed, anecdotal reports have circulated for years among thoracic surgeons about patients with severe emphysema who believed they were able to breathe better after a lobectomy, usually of an upper lobe, done because of lung cancer. Patients with significant emphysema and cancer located in the areas of best-preserved lung, however, would not be candidates for a lobectomy. In this circumstance one might consider a wedge or segmental excision of the cancer concomitant with LVR. Although this approach leads to an increased risk of local recurrence, in suitable candidates it likely would leave the patient with a better respiratory status and a better chance of cure than would primary radiation therapy. Radiation therapy, in nearly all circumstances, will leave the patient with worse pulmonary function than they had at the onset of treatment. Surgical resection, in appropriate candidates, not only offers the best chance for cure but is also the only treatment modality with the potential to improve the patients' respiratory status after therapy. As such, operation should be considered in patients with an appropriate anatomic situation regardless of the degree of impairment in measured PFTs. In this series, patients were carefully screened for the presence of comorbid conditions. Likely, the presence of nonpulmonary organ dysfunction would greatly increase the operative risks in these patients and should be factored into the decision on how to treat the lung cancer.

A number of issues remain unresolved. It has been our policy not to consider LVR in any patient who has smoked cigarettes within the previous 6 months. Furthermore, we insist on a period of preoperative pulmonary rehabilitation, usually for 8 weeks, with the goal of maximizing exercise endurance and capacity. To what extent these requirements should be altered in patients with coexisting lung cancer remains undetermined. Review of our experience with structured pulmonary rehabilitation in patients undergoing LVR has shown that with preoperative rehabilitation patients improve their 6-minute walk distance an average of $30 \%$ to $40 \%$ over the distance at the time of evaluation. In this group of five patients, four had preoperative rehabilitation, and two of these patients demonstrated improvement in 6-minute walk distance. One patient was found to be severely deconditioned at evaluation and with 8 weeks of rehabilitation increased his 6-minute walk distance by $2725 \%$ (from 40 feet to 1090 feet). Clearly, patients in the worst cardiorespiratory condition at the time of evaluation stand to gain the most with preoperative rehabilitation, whereas the gains in those in better condition initially are likely to be marginal. Indeed, one of the five patients in this report was judged at evaluation to be fit and did well without any preoperative rehabilitation. Furthermore, the two patients who had 6-minute walk distances greater than 1000 feet at evaluation did not show any improvement with structured pulmonary rehabilitation. Thus delay in resection for rehabilitation is probably only justified in the most deconditioned patients, and perhaps the 6-minute walk test might prove to be a helpful discriminator to determine who might benefit most from preoperative structured pulmonary rehabilitation. Currently, however, a minimum of several weeks of smoking cessation and exercise rehabilitation would, in our opinion, seem essential in many of these patients.

In summary, we believe that the introduction of volume reduction operations has added a new arm in the algorithm for the evaluation and treatment of lung cancer in patients with advanced emphysema. In this report we have demonstrated that within the group of patients with both emphysema and lung cancer there exists a subset of patients whose disease is anatomically suitable for a combined lobectomy and volume reduction. This combination not only allows an optimal cancer resection in patients who might otherwise be considered inoperable, but also in appropriately selected patients results in improved postoperative subjective and objective pulmonary function. Thus patients with lung cancer and poor PFT findings should not automatically be labeled as unfit for resection. Rather, given the propensity for both emphysema and lung cancer to occur in patients with a significant smoking history, it is likely that a combined approach could be applicable to a significant number of patients. Undoubtedly, further experience with LVR operations, both alone and in combination with a cancer operation, will help refine the indications, contraindications, and limitations of such an approach. 
We acknowledge the invaluable assistance in preparation of the data for this manuscript provided by Ms. Roni Richardson Higgins, RN.

\section{REFERENCES}

1. Ginsberg RJ, Rubinstein LV, Lung Cancer Study Group. Randomized trial of lobectomy versus limited resection for T1 N0 non-small cell lung cancer. Ann Thorac Surg 1995;60: 615-23.

2. Kaskowitz L, Graham MV, Emaini B, Halverson KJ, Rush C. Radiation therapy alone for stage I non-small cell lung cancer. Int J Radiat Oncol Biol Phys 1993;27:517-23.

3. Murren JR, Buzaid AC. Chemotherapy and radiation for the treatment of non-small-cell lung cancer: a critical review. Clin Chest Med 1993;14:161-71.

4. Bezjak A, Payne D. Radiotherapy in the management of non-small-cell lung cancer. World J Surg 1993;17:741-50.

5. Cooper JD, Trulock EP, Triantafillou AN, et al. Bilateral pneumectomy (volume reduction) for chronic obstructive pulmonary disease. J Thorac Cardiovasc Surg 1995;109:10619 .

6. Cooper JD, Patterson GA, Sundaresan RS, et al. Results of 150 consecutive bilateral lung volume reduction procedures in patients with severe emphysema. J Thorac Cardiovasc Surg 1996;112:1319-30.

7. Vial WC. Southwestern Internal Medicine Conference: cigarette smoking and lung disease. Am J Med Sci 1986;291: 130-42.

8. Samet JM. The epidemiology of lung cancer. Chest 1993;103: 20s-9s.

9. Pigula FA, Keenan RJ, Ferson PF, Landreneau RJ. Unsuspected lung cancer found in work-up for lung reduction operation. Ann Thorac Surg 1996;61:174-6.

10. Boushy SF, Billig DM, North LB, Helgason AH. Clinical course related to preoperative and postoperative pulmonary function in patients with bronchogenic carcinoma. Chest 1971;59:383-91.

11. Miller JI, Grossman GD, Hatcher CR. Pulmonary function test criteria for operability and pulmonary resection. Surg Gynecol Obstet 1981;153:893-5.

12. Miller JE. Physiologic evaluation of pulmonary function in the candidate for lung resection. J Thorac Cardiovasc Surg 1993; 105:347-52.

13. Ferguson MK, Little L, Rizzo L, et al. Diffusing capacity predicts morbidity and mortality after pulmonary resection. J Thorac Cardiovasc Surg 1988;96:894-90.

14. Olsen GN, Block J. Pulmonary function testing in evaluation for pneumonectomy. Hosp Pract 1973;8:137-44.

15. Kristersson S, Arborelius M, Jungquist G, et al. Prediction of ventilatory capacity after lobectomy. Scand J Respir Dis 1973;54:315-25.

16. Ali MK, Mountain CF, Ewer MS, et al. Predicting loss of pulmonary function after pulmonary resection for bronchogenic carcinoma. Chest 1980;77:337-42.

17. Wernly JA, DeMeester TR, Kirchner PT, et al. Clinical value of quantitative ventilation-perfusion lung scans in the surgical management of bronchogenic carcinoma. J Thorac Cardiovasc Surg 1980;80:535-43.

18. Gaissert HA, Trulock EP, Cooper JD, Sundaresan SR, Patterson GA. Comparison of early results after volume reduction or lung transplantation for chronic obstructive pulmonary disease. J Thorac Cardiovasc Surg 1996;111:296-307.

19. Brantigan OC, Kress MB, Mueller EA. The surgical approach to pulmonary emphysema. Chest 1961;39:485501.

20. Keenan RJ, Landreneau RJ, Sciurba FC, et al. Unilateral thoracoscopic surgical approach for diffuse emphysema. J Thorac Cardiovasc Surg 1996;111:308-16. 